\title{
EQUILIBRIA IN REFLEXIVE BANACH LATTICES WITH A CONTINUUM OF AGENTS
}

\author{
A. ARAUJO, V. F. MARTINS-DA-ROCHA AND P. K. MONTEIRO
}

\begin{abstract}
We consider exchange economies with a measure space of agents and for which the commodity space is a separable and reflexive Banach lattice. Under assumptions imposing uniform bounds on marginal rates of substitution, positive results on core-Walras equivalence were established in Rustichini-Yannelis [27] and Podczeck [25]. In this paper we prove that under similar assumptions on marginal rates of substitution, the set of competitive equilibria (and thus the core) is non-empty.
\end{abstract}

\section{INTRODUCTION}

We consider an exchange economy with infinitely many agents and infinitely many commodities. Infinite dimensional commodity spaces arise very naturally in economics, in particular in problems involving the allocation of resources over an infinite time horizon (e.g. an $\ell^{p}$ commodity space) or uncertainty about the possibly infinite number of states of nature (e.g. an $L^{p}([0,1])$ commodity space). In our model, the commodity space we will be a reflexive and separable Banach space. In the formulation of the Arrow-Debreu-McKenzie model of an exchange economy (Arrow-Debreu [5], McKenzie [22], Debreu [11]), a finite number of agents take prices as given. This formulation raises a conceptual difficulty: a finite number of agents should mean that individuals are able to exercise some influence, which contradicts the price-taking behavior assumption. To model perfectly competitive markets, we follow Aumann [6,7] and Hildenbrand [14], who suggested to model the set of agents by a finite complete measure space. The insignificance of individual agents is thus captured by the idea of a set of zero measure.

In the literature dealing with large economies (infinitely many agents), two solution concepts are used: the competitive (Walrasian) equilibrium and the core. For the first concept, agents are assumed to take prices as given and they engage in the sale and purchase of commodities in order to maximize their utilities subject to their budgets. Agents trade freely in a decentralized market and this process results in allocations which equate supply with demand. The second concept allows for the possibility of cooperation among agents. They are allowed to bargain multilaterally which leads to an allocation of resources where it is not possible for any coalition of agents to redistribute their initial endowments among themselves in any way that makes each member of the coalition better off. Aumann [6] proved that in perfectly competitive economies (i.e. economies with an atomless finite measure space of agents) with finitely many commodities, the core coincides with the set of competitive equilibria. He also proved in [7], that the set of competitive equilibria (and thus the core) is non-empty. The core-Walras equivalence theorem was extended by RustichiniYannelis [27], to commodity spaces being separable Banach spaces.

Date: January 22, 2004. 
In the framework of large square economies (i.e. with infinitely many agents and infinitely many commodities), there are several equilibrium existence results: Bewley [9], Khan-Yannelis [16], Podczeck [24] and Martins-da-Rocha [19] for separable Banach commodity spaces with an interior point in the positive cone; and Mas-Colell [21], Jones [15], Ostroy-Zame [23], Podczeck [24, 26] and Martins-da-Rocha [20] for economies with differentiated commodities. ${ }^{1}$ To the best of our knowledge, this paper is the first to provide an equilibrium existence result for economies with a separable and reflexive Banach lattice (i.e. $\ell^{p}$ or $L^{p}([0,1])$ for $\left.1<p<+\infty\right)$. Under assumptions imposing uniform bounds on marginal rates of substitution, positive results on core-Walras equivalence were established in Rustichini-Yannelis [27] and Podczeck [25]. In this paper we prove that under similar assumptions on marginal rates of substitution, the set of competitive equilibria (and thus the core) is non-empty. More precisely, we provide two frameworks to prove the existence of competitive equilibria. In the first one, existence is proved under an assumption (borrowed from Zame [30] and Podczeck [25]) imposing the existence, at each state of nature, of uniform (over consumption) upper and lower bounds on marginal rates of substitution. In the second one, the commodity space is $\ell^{p}$ and existence is proved for preference relations represented by separable utility functions. But for this framework, we only require the existence of an upper bound on the marginal rates of substitution at the initial endowment (and not uniformly over consumption) and we require the existence of a uniform (over consumption) lower bound on the marginal rates of substitution at only one state of nature.

Recently, Tourky-Yannelis [28] showed that, when aggregation of individual commodity bundles is formalized in terms of the Bochner integral, given a non-separable Hilbert space $E$, and given any atomless measure space $(\Omega, \mathcal{A}, \mu)$, there is an economy with $(\Omega, \mathcal{A}, \mu)$ as space of agents and $E$ as commodity space that has a non-empty core but does not have a competitive equilibrium. Contrasting with the positive results of Aumann $[6,7]$ and their extensions to separable Banach commodity spaces by Rustichini-Yannelis [27], the crucial condition to get these results is that there are "many more agents than commodities". More precisely, Podczeck [25] proved that the class $\{E\}$ of Banach spaces such that, under a list of "desirable assumptions", any atomless economy with commodity space $E$ exhibits core-Walras equivalence ${ }^{2}$ is exactly the class of Banach spaces that are separable. However, there is no characterization in the literature of a class of Banach spaces as those spaces in which the existence of competitive equilibria holds. In our framework, the commodity space is a separable Banach space, thus under "desirable assumptions" the core-Walras equivalence theorem is valid. But, the separability assumption of the commodity space is no more sufficient to get the existence of competitive equilibria. We introduce an additional assumption which requires a compatibility (Definition 2.2 and Assumption 3.4) between the geometry of the lattice ordering of the commodity space and initial endowments. When $L^{2}([0,1])$ is endowed with the natural pointwise lattice ordering, we construct an economy satisfying a list of "desirable assumptions", but not satisfying the compatibility assumption and for which there are no competitive equilibria at all. It appears that for the issue of the existence of competitive equilibria, the topological way of measuring the cardinality of the number of markets introduced by Tourky-Yannelis [28] is not appropriate. It is the geometric structure of the lattice ordering that matters. In this paper, several examples of lattice ordering which satisfy the compatibility condition are given. For these examples, the positive cone has countably many extreme rays. However the number of extreme

\footnotetext{
${ }^{1}$ The commodity space is $M([0,1])$ the space of Radon measures on $[0,1]$ and the price space is $C([0,1])$ the space of continuous functions on $[0,1]$.

${ }^{2}$ When feasibility of allocation is defined in terms of the Bochner integral.
} 
directions of the positive cone is not the appropriate way of measuring the cardinality of the number of markets since positive results for smooth positive cones ${ }^{3}$ are given in Martins-da-Rocha [20].

Following the approach used in Martins-da-Rocha [19, 20], our proof of the existence of an equilibrium is based on the discretization of the set of agents. We approximate the initial economy $\mathcal{E}$ by a sequence of economies $\left(\mathcal{E}^{n}\right)$ with finitely many agents. To each finite economy $\mathcal{E}^{n}$, we use the lattice structure of the commodity space and the properness assumptions on preferences to get the existence of a quasi-equilibrium $\left(x^{n}, p^{n}\right)$. The last step consists on proving that the sequence $\left(x^{n}, p^{n}\right)$ converges to an equilibrium $(x, p)$. In order to apply a Fatou type lemma to the sequence of mappings $\left(x^{n}\right)$, we need a specific compatibility (Definition 2.2 and Assumption 3.4) between the geometry of the lattice ordering and initial endowments.

The paper is organized as follows. In Section 2 we define the model of an economy with infinitely many agents and commodities and we set out the main definitions and notations. In Section 3 we give the list of assumptions that economies will be required to satisfy and we present the two existence results. The different assumptions on the marginal rates of substitution are discussed in Section 4. Finally, Section 5 is devoted to the proof of the two theorems.

\section{The Model}

2.1. Preliminaries. Let $E$ be a separable and reflexive Banach lattice. ${ }^{4}$ We denote by $E^{*}$ the dual space of $E$, i.e. the space of all continuous linear functions from $E$ into $\mathbb{R}$. If $x \in E$ and $p \in E^{*}$, the value $p(x)$ of $p$ at $x$ will often be denoted $\langle p, x\rangle$. We write $\|$.$\| for both the norm of E$ and the dual norm of $E^{*}$. We write $w$ for the weak topology $\sigma\left(E, E^{*}\right)$ on $E, w^{*}$ for the weak-star topology $\sigma\left(E^{*}, E\right)$ on $E^{*}$, and $s$ for the norm-topology. As usual, the ordering of $E$ is denoted by $\geqslant$, and $E_{+}$denotes the positive cone of $E$, i.e. $E_{+}=\{x \in E: x \geqslant 0\}$. The dual space $E^{*}$ will always be regarded as endowed with the dual ordering, i.e. $E_{+}^{*}=\left\{p \in E^{*}: p(x) \geqslant 0, \forall x \in E_{+}\right\}$. A vector $x \in E$ is said positive if $x \geqslant 0$, a linear functional $q \in E^{*}$ is said strictly positive if $q(x)>0$ whenever $x$ belongs to $E_{+} \backslash\{0\}$. For $x, y \in E$ the expressions $x^{+}, x^{-},|x|$ have the usual lattice theoretical meaning. Let $\tau$ be a topology on $E$. If $\left(C_{n}\right)_{n}$ is a sequence of subsets of $E$, the $\tau$-sequential upper limit of $\left(C_{n}\right)_{n}$, is denoted $\tau$ - $\operatorname{ls}_{n} C_{n}$ and is defined by

$$
\tau-\operatorname{ls}_{n} C_{n}:=\left\{x \in E: x=\tau \text { - } \lim _{k} x^{k}, \quad x^{k} \in C_{n(k)}\right\}
$$

where $\left(C_{n(k)}\right)_{k}$ is a subsequence of $\left(C_{n}\right)_{n}$.

The Borel $\sigma$-algebra of $E$ for the norm-topology or for the weak-topology coincide and is denoted by $\mathcal{B}$. Let $(\Omega, \mathcal{A}, \mu)$ be a complete finite positive measure space. A correspondence $F$ from $\Omega$ to $E$ is said to be graph measurable if $\left\{(a, x) \in \Omega \times \ell^{p}: x \in F(a)\right\}$ belongs to $\mathcal{A} \otimes \mathcal{B}$. A correspondence $P$ from $\Omega$ to $E \times E$ is said to be graph measurable if $\{(a, x, z) \in \Omega \times E \times E:(x, z) \in P(a)\}$ belongs to $\mathcal{A} \otimes \mathcal{B} \otimes \mathcal{B}$. A mapping $s$ from $\Omega$ to $E$ is simple if there exist $x_{1}, x_{2}, \ldots, x_{n}$ in $E$ and $A_{1}, A_{2}, \ldots, A_{n}$ in $\mathcal{A}$ such that $s=\sum_{i=1}^{n} x_{i} \chi_{A_{i}}$ where $\chi_{A_{i}}(a)=1$ if $a \in A_{i}$ and $\chi_{A_{i}}(a)=0$ if $a \notin A_{i}$. A mapping $x: \Omega \rightarrow E$ is Bochner measurable if there is a sequence of simple mappings $s_{n}: \Omega \rightarrow E$ such that $\lim _{n}\left\|s_{n}(a)-x(a)\right\|=0$ almost every where. Since $E$ is separable, we know from Pettis' measurability theorem (see [12, Theorem II.1.2, p.42]) that a mapping $x: \Omega \rightarrow E$ is Bochner measurable if and only if for each $B \in \mathcal{B}, x^{-1}(B):=\{a \in \Omega: x(a) \in B\}$ belongs to $\mathcal{A}$. A Bochner measurable mapping $x$ from $\Omega$ to $E$ is Bochner integrable if there is a sequence of simple mappings $s_{n}: \Omega \rightarrow E$ such that $\lim _{n} \int_{\Omega}\left\|s_{n}(a)-x(a)\right\| d \mu(a)=0$. For each measurable set

\footnotetext{
${ }^{3}$ The commodity space is $M([0,1])$ ordered by the natural pointwise positive cone.

${ }^{4}$ We refer to Aliprantis-Border [2] for definitions.
} 


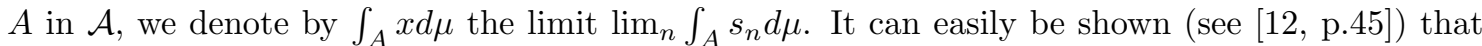
a Bochner measurable mapping $x$ is Bochner integrable if and only if the mapping $a \mapsto\|x(a)\|$ is integrable. In particular $\left\|\int_{A} x d \mu\right\| \leqslant \int_{A}\|x(a)\| d \mu(a)$.

2.2. Fatou's cone. We define hereafter a class of lattice orderings which will enable us to apply a Fatou type lemma.

Definition 2.1. Let $E$ be a Banach lattice, a functional $\rho$ from $E$ into $[-\infty,+\infty]$ is a positive extended linear functional if

(i) the space $E^{\rho}=\{x \in E: \rho(x) \in \mathbb{R}\}$ is a vector subspace of $E$,

(ii) the restriction of $\rho$ to $E^{\rho}$ is linear and,

(iii) the functional $\rho$ is positive, i.e. for any $y \geqslant x \geqslant 0$, we have $\rho(y) \geqslant \rho(x) \geqslant 0$.

Definition 2.2. Let $E$ be a Banach lattice ordered by a positive cone $E_{+}$and let $e: \Omega \rightarrow E_{+}$ be a Bochner integrable mapping. The cone $E_{+}$is a Fatou's cone relatively to $e$ if there exists a positive extended linear functional $\rho$ such that

(a) for every $x$ in $E_{+},\|x\| \leqslant \rho(x)$,

(b) the function $a \mapsto \rho[e(a)]$ from $\Omega$ to $\mathbb{R}_{+}$is integrable.

Example 2.3. Take $E=\ell^{p}$ for any $1<p<+\infty$, and $E_{+}=\ell_{+}^{p}$ the natural pointwise positive cone. $^{5}$ Then, for any Bochner integrable mapping $e: \Omega \rightarrow \ell_{+}^{p}$, if the function ${ }^{6} a \mapsto\|e(a)\|_{1}$ is integrable, then $E_{+}$is a Fatou's cone relatively to $e$.

Example 2.4. Take $E=L^{2}([0,1])$ and let $\left(b_{n}\right)_{n}$ be an Hilbert basis of $E$. Let

$$
E_{+}=\left\{x \in E: \forall n \in \mathbb{N},\left\langle x, b_{n}\right\rangle \geqslant 0\right\},
$$

then $E$ is a Banach lattice. Moreover, if $e: \Omega \rightarrow E_{+}$is a Bochner integrable mapping such that the function

$$
a \longmapsto \sum_{n}\left\langle e(a), b_{n}\right\rangle
$$

is integrable, then $E_{+}$is a Fatou's cone relatively to $e$.

Remark 2.5. In the above two examples, the positive cone has countably many extreme rays. Let $(T, \mathcal{T}, \sigma)$ be an atomless measure space and $1<p<+\infty$. We prove in Appendix $\mathrm{D}$ that when $E=L^{p}(T, \mathcal{T}, \sigma)$ is ordered by the pointwise "smooth" positive cone $E_{+}=L^{p}(T, \mathcal{T}, \sigma)_{+}$, then for every Bochner integrable mapping $e: \Omega \rightarrow E_{+}$with $\int_{\Omega} e d \mu>0$, the cone $E_{+}$is not a Fatou's cone relatively to $e$.

2.3. The Model. An economy $\mathcal{E}$ is a list

$$
\mathcal{E}=((\Omega, \mathcal{A}, \mu), E, X, \succeq, e),
$$

where $X$ is a correspondence from $\Omega$ to $E, \succeq$ is a correspondence from $\Omega$ to $E \times E$ and $e$ is a mapping from $\Omega$ to $E$. The space of agents is $(\Omega, \mathcal{A}, \mu)$, a complete finite positive measure space. The commodity space is $E$. For each agent $a \in \Omega$, the consumption set is $X(a)$, the initial endowment is $e(a) \in E$ and the preference/indifference relation is $\succeq_{a} \subset X(a) \times X(a)$, a reflexive binary relation on $X(a)$.

\footnotetext{
${ }^{5}$ The natural pointwise positive cone of $\ell^{p}$ is $\ell_{+}^{p}=\left\{x=\left(x_{n}\right) \in \ell^{p}: \forall n \in N, x_{n} \geqslant 0\right\}$.

${ }^{6}$ If $x=\left(x_{n}\right)$ belongs to $\ell^{p}$, we let $\|x\|_{1}=\sum_{n}\left|x_{n}\right|$.
} 
We define the correspondence ${ }^{7} P_{a}: X(a) \rightarrow X(a)$ by $P_{a}(x)=\left\{x^{\prime} \in X(a): x^{\prime} \succ_{a} x\right\}$. In particular, if $x \in X(a)$ is a consumption bundle, the set $P_{a}(x)$ is the set of consumption bundles strictly preferred to $x$ by agent $a$. We let $P$ be the correspondence from $\Omega$ to $E \times E$ defined for each $a \in \Omega$ by $P(a)=\left\{\left(x, x^{\prime}\right) \in X(a) \times X(a): x^{\prime} \succ_{a} x\right\}$.

The set of allocations (or plans) of the economy is the set $S^{1}(X)$ of Bochner integrable selections of $X$, i.e. $S^{1}(X)$ is the set of mappings $x$ from $\Omega$ to $E$ which are Bochner integrable and which satisfies $x(a) \in X(a)$ for almost every $a \in \Omega$. An allocation $x \in S^{1}(X)$ is feasible if

$$
\int_{\Omega} x d \mu=\int_{\Omega} e d \mu
$$

We assume that the mapping $e: \Omega \rightarrow E$ is a Bochner integrable mapping and we denote by $\omega:=\int_{\Omega} e d \mu$ the aggregate initial endowment.

Definition 2.6. A pair $(x, p)$ consisting of a feasible allocation $x$ and a non-zero price $p$ is said to be a competitive equilibrium if for almost every $a \in \Omega,\langle p, x(a)\rangle=\langle p, e(a)\rangle$, and $z \in P_{a}(x(a))$ implies $\langle p, z\rangle>\langle p, x(a)\rangle$.

\section{Existence OF A COMPetitive Equilibrium}

We will maintain in this paper the following assumptions on the economy $\mathcal{E}$.

Assumption 3.1. For each $a \in \Omega$,

(i) the consumption set is $X(a)=E_{+}$;

(ii) the initial endowment is not zero, i.e. $e(a)>0$;

(iii) $\succeq_{a}$ is reflexive, transitive and complete;

(iv) $\succeq_{a}$ is strictly monotone, i.e. for each $x \in X(a)$, if $z>x$ then $z \succ_{a} x$.

Assumption 3.2. For each $a \in \Omega$, for every $x \in X(a)$,

(i) the sets $P_{a}(x)$ and $P_{a}^{-1}(x)=\left\{z \in X(a): x \succ_{a} z\right\}$ are norm-open in $X(a)$;

(ii) the set $\left\{z \in X(a): z \succeq_{a} x\right\}$ is convex.

Assumption 3.3. The correspondence $P$ is graph measurable.

Assumption 3.4. The positive cone $E_{+}$is a Fatou's cone relatively to $e$.

Remark 3.1. Assumptions 3.1-3.3 are standard in the literature dealing with exchange economies with finitely or infinitely many agents. We will see that in our framework, we can not dispense with Assumption 3.4.

We provide hereafter two frameworks to prove the existence of competitive equilibria. In the first one, existence is proved under an assumption imposing upper and lower uniform (over agents and consumption) bounds on marginal rates of substitution. In the second one, existence is proved for preference relations represented by separable utility functions defined on $\ell^{p}$, but the assumption on the marginal rates of substitution required for the existence is weaker. We only require a uniform (over agents only) upper bound on the marginal rates of substitution at the initial endowment and a uniform (over agents and consumption) lower bound on the marginal rates of substitution at only one state of nature.

\footnotetext{
${ }^{7}$ As usual, $y \succ_{a} x$ means $\left[y \succeq_{a} x\right.$ and $\left.x \succeq_{a} y\right]$. Note that the binary relation $\succ_{a}$ coincide with the graph of the correspondence $P_{a}$.
} 
3.1. The general case. In this section, we consider economies with general preference relations. The following requirement is borrowed from Zame [30]. It is discussed in Section 4.

Definition 3.2. The preference relations $\left(\succeq_{a}\right)$ are said to be strong-uniformly proper, if there exist strictly positive prices $\alpha$ and $\beta$ in $E^{*}$ with $\alpha \leqslant \beta$ and such that for every $a \in \Omega$, whenever $x, u, v \in E_{+}$satisfy $v \leqslant x$ and $\langle\alpha, u\rangle>\langle\beta, v\rangle$ then $x-v+u \succ_{a} x$.

An economy $\mathcal{E}$ is said strong-uniformly proper if it has strong-uniformly proper preference relations.

We can now state our first result for economies with general preference relations.

Theorem 3.3. If the economy $\mathcal{E}$ is strong-uniformly proper then there exists a competitive equilibrium.

Remark 3.4. The strong-uniform properness assumption was already used in Zame [30]. Podczeck in [26] proved the equivalence between the core and the set of competitive equilibria under this assumption. Note that Rustichini-Yannelis [27] also proved the equivalence between the core and the set of competitive equilibria under another properness assumption.

Assumption 3.4 is unusual. Following Zame [30] we provide hereafter two examples of a stronguniformly proper economies satisfying Assumptions 3.1-3.3 and not satisfying Assumption 3.4. For these economies the set of competitive equilibria is empty.

Counterexample 3.5. Consider the economy $\mathcal{E}$ where $\Omega=[0,1], \mathcal{A}$ is the Lebesgue $\sigma$-algebra and $\mu$ is the Lebesgue measure. The commodity space $E$ is $\ell^{p}$ for $1<p<+\infty$, ordered by the pointwise positive cone $\ell_{+}^{p}$. For each trader $a \in[0,1]$, the utility function $u_{a}$ is defined by

$$
u_{a}(x)=\sum_{n \in \mathbb{N}}(2+a)^{-n} x_{n}
$$

and the initial endowment is defined by $e(a)=(1,1 / 2,1 / 3, \ldots, 1 / n, \ldots)$. The economy $\mathcal{E}$ is strong-uniformly proper, it satisfies Assumptions 3.1-3.3 but not Assumption 3.4. It is proved in Zame [30] that $\mathcal{E}$ has no competitive equilibrium.

Counterexample 3.6. Consider the economy $\mathcal{E}$ where $\Omega=[0,1], \mathcal{A}$ is the Lebesgue $\sigma$-algebra and $\mu$ is the Lebesgue measure. The commodity space $E$ is $L^{p}([0,1], \mu)$ where $1<p<+\infty$ ordered by the "smooth" pointwise positive cone $L^{p}([0,1], \mu)_{+}$For each trader $a \in[0,1]$, the utility function $u_{a}$ is defined by

$$
u_{a}(x)=\int_{[0,1]} q_{a}(t) x(t) \mu(d t)
$$

and the initial endowment is $e(a): t \mapsto 1$. For each $a \in(0,1]$, the function $q_{a}$ is defined by

$$
q_{a}(t)= \begin{cases}\frac{1}{2}+\frac{t}{2 a} & \text { if } 0 \leqslant t \leqslant a \\ \frac{a-2}{2(a-1)}+\frac{t}{2(a-1)} & \text { if } a \leqslant t \leqslant 1 .\end{cases}
$$

The economy $\mathcal{E}$ is strong-uniformly proper, it satisfies Assumptions 3.1-3.3 but not Assumption 3.4. It is proved in Zame [30] that $\mathcal{E}$ has no competitive equilibrium. 
3.2. The separable case. In this section, we consider economies with the space $\ell^{p}$ as the commodity space and for which preference relations are represented by separable utility functions. For each $1<p<+\infty$, we denote by $\ell^{p}$ the real vector space of sequences $x=\left(x_{k}\right)_{k}$ in $\mathbb{R}^{\mathbb{N}}$ such that $\lim _{n} \sum_{k=0}^{n}\left|x_{k}\right|^{p}<\infty$ and we denote by $\|x\|_{p}=\left(\sum_{k \in \mathbb{N}}\left|x_{k}\right|^{p}\right)^{1 / p}$. We denote by $\ell_{+}^{p}$ the natural positive cone defined by $x \in \ell_{+}^{p}$ if and only if $x_{k} \geqslant 0$ for each $k \in \mathbb{N}$. The space $\ell^{p}$ endowed with the norm $\|\cdot\|_{p}$ and the positive cone $\ell_{+}^{p}$ is a reflexive and separable Banach lattice whose dual is $\ell^{q}$ where $1<q<+\infty$ is defined by $1 / p+1 / q=1$.

Definition 3.7. A utility function $u: \ell_{+}^{p} \rightarrow \mathbb{R}$ is called separable if there exists for each $n$, a function $v_{n}:[0,+\infty) \rightarrow \mathbb{R}$ concave and strictly increasing such that

$$
\forall x \in \ell_{+}^{p}, \quad u(x)=\sum_{n \in \mathbb{N}} v_{n}\left(x_{n}\right) .
$$

The function $v=\left(v_{n}\right)$ is called the kernel of $u$. The left derivative of $v_{n}$ in $t>0$ is noted $v_{n}^{-}(t)$ and the right derivative is denoted $v_{n}^{+}(t)$. If $x \in \ell_{+}^{p}$ then we note $v^{-}(x):=\left(v_{n}^{-}\left(x_{n}\right)\right)_{n}$. For $x \in \ell_{+}^{p}$ we define $S(x)=\left\{h \in \ell^{p}: \exists t>0, x+t h \geqslant 0\right\}$ and $I(x)=S(x) \cap-S(x)$. We define $u^{\prime}(x) \cdot h=\lim _{r \rightarrow 0}(1 / r)[u(x+r h)-u(x)]$ for each $x \in \ell_{+}^{p}$ and each $h \in S(x)$. Note that if $h \geqslant 0$ then $u^{\prime}(x) \cdot h=\sum_{n} v_{n}^{+}\left(x_{n}\right) h_{n}$.

For economies with separable utility functions, a weaker condition than the uniform properness will be sufficient to prove the existence of competitive equilibria.

Definition 3.8. An economy $\mathcal{E}$ is said separably proper if for each agent $a \in \Omega$, the preference relation $\succ_{a}$ is represented ${ }^{8}$ by a separable utility function $u_{a}$ which kernel is denoted $v_{a}$ and if there exists a measurable set $\Omega^{\prime} \in \mathcal{A}$ of full measure, ${ }^{9}$ satisfying the following conditions.

(a) There exists $\beta \in \ell^{q}$ such that for each $a \in \Omega^{\prime}$,

$$
v_{a}^{-}(e(a)) \leqslant \beta .
$$

(b) There exists $k \in \mathbb{N}$ and $\alpha_{k}>0$ such that $\omega_{k} \alpha_{k}>0$ and for each $a \in \Omega^{\prime}$,

$$
0<\alpha_{k} \leqslant \inf \left\{v_{a, k}^{+}(t): t \geqslant 0\right\}=\lim _{t \rightarrow+\infty} v_{a, k}^{+}(t) .
$$

We can now state our second existence result for economies with preference relations represented by utility functions.

Theorem 3.9. If the economy $\mathcal{E}$ is separably proper then there exists a competitive equilibrium.

The two properness conditions are not comparable. Obviously, not all strong-uniformly proper economies are separably proper. Moreover, we provide hereafter an example of an economy which is separably proper but not strong-uniformly proper.

Example 3.10. Consider the economy $\mathcal{E}$ where $\Omega=[0,1], \mathcal{A}$ is the Lebesgue $\sigma$-algebra and $\mu$ is the Lebesgue measure. For each trader $a \in[0,1]$, the consumption set coincide with $\ell_{+}^{p}$, the utility function $u_{a}$ is defined by

$$
u_{a}(x)=x_{0}+\sum_{n \geqslant 1} \frac{1-\exp \left(-a x_{n} n^{2}\right)}{n^{2}}
$$

${ }^{8}$ That is $x^{\prime} \succ_{a} x$ if and only if $u_{a}\left(x^{\prime}\right)>u_{a}(x)$.

${ }^{9}$ That is $\mu\left(\Omega \backslash \Omega^{\prime}\right)=0$. 
and the initial endowment is defined by $e(a)=(1,1 / 2,1 / 3, \ldots, 1 / n, \ldots)$. Following Example 4.5 the economy $\mathcal{E}$ satisfies Assumptions 3.1-3.4. Moreover this economy is separably proper but not strong-uniformly proper.

\section{Proper ECONOMies}

We discuss in this section the notions of properness used in Theorem 3.3 and Theorem 3.9.

4.1. Strong-uniformly proper economies. We recall that the preference relations $\left(\succeq_{a}\right)$ are said to be strong-uniformly proper, if there exist strictly positive prices $\alpha$ and $\beta$ in $E_{+}$with $\alpha \leqslant \beta$ and such that for every $a \in \Omega$, whenever $x, u, v \in E_{+}$satisfy $v \leqslant x$ and $\langle\alpha, u\rangle>\langle\beta, v\rangle$ then $x-v+u \succ_{a} x$. This properness condition is borrowed from Zame [30]. Note that this is a requirement on preferences that is uniform over agents as well as over consumption. We refer to Zame [30] for a discussion of this condition as well as for corresponding examples. Following Podczeck [25], it may be seen that if for each $a \in \Omega,\left\{y \in X(a): y \succeq_{a} x\right\}$ is convex then uniform properness is equivalent to the following statement: There are strictly positive prices $\alpha, \beta \in E^{*}$, such that given any $a \in \Omega$ and $x \in X(a)$ there is a price $p$ in the order interval $[\alpha, \beta]$ such that $\langle p, x\rangle \leqslant\langle p, y\rangle$ for all $y \in X(a)$ with $y \succeq_{a} x$. Since supporting prices are measures of marginal rates of substitution, the strong-uniform properness assumption is a condition that puts strong bounds on these rates.

We recall the notion of uniform properness introduced by Yannelis-Zame [29] for economies with finitely many agents.

Definition 4.1. The preference relations $\left(\succeq_{a}\right)$ are said to be $v$-uniformly proper with $v \in E$, if there exists a norm-open 0-neighborhood $V \subset E$ such that for each $a \in \Omega$, for each $x \in E_{+}$, $(x+\Gamma) \cap E_{+} \subset P_{a}(x)$ where $\Gamma=\cup_{t>0} t(v+V)$

Remark 4.2. The strong-uniform properness assumption on the preference relations implies that

$$
\forall a \in \Omega, \quad \forall x \in E_{+}, \quad(x+\Gamma) \cap E_{+} \subset P_{a}(x),
$$

where $\Gamma$ is the convex and norm-open cone defined by $\Gamma=\left\{x \in E: \alpha\left(x^{+}\right)>\beta\left(x^{-}\right)\right\}$.

Example 4.3. Consider the case of positive separable utility functions $u_{a}: \ell_{+}^{p} \rightarrow \mathbb{R}$, defined by the formula $u_{a}(x)=\sum_{n} v_{a, n}\left(x_{n}\right)$ where for each $n$, the function $v_{a, n}:[0,+\infty) \rightarrow \mathbb{R}$ is continuous, the derivative $v_{a, n}^{\prime}(t)$ exists for each $t>0$. Suppose that there exist $\alpha$ and $\beta$ two strictly positive functionals in $\ell^{q}$ such that

$$
\forall a \in \Omega, \quad \forall t>0, \quad \alpha_{n} \leqslant v_{a, n}^{\prime}(t) \leqslant \beta_{n} .
$$

Then the preference relations defined by the utility functions $\left(u_{a}\right)_{a \in \Omega}$ are strong-uniformly proper. Indeed, let $x, y, z \in \ell_{+}^{p}$ satisfying $y \leqslant x$ and $\alpha(z)>\beta(y)$. Using the mean value theorem, we see that for each $n$ there exists $t_{n}>0$ such that

$$
v_{a, n}\left(x_{n}-y_{n}+z_{n}\right)-v_{n}(x)=v_{a, n}^{\prime}\left(t_{n}\right)\left[z_{n}-y_{n}\right] .
$$

But $v_{a, n}^{\prime}\left(t_{n}\right)\left[z_{n}-y_{n}\right] \geqslant \alpha_{n} z_{n}-\beta_{n} y_{n}$, in particular

$$
u(x-y+z)-v(x) \geqslant \alpha(z)-\beta(y)>0 .
$$

We refer to Araujo-Monteiro [3], Le Van [17] and Aliprantis [1] for precisions about proper conditions for separable utility functions. 
4.2. Separably proper economies. Following Aliprantis [1], we introduce the following notion of separable utility function.

Definition 4.4. A separable utility function $u: \ell_{+}^{p} \rightarrow \mathbb{R}$, where $u(x)=\sum_{n} v_{n}\left(x_{n}\right)$, is said to be rational if for each $n \in \mathbb{N}$,

(a) $v_{n}(0)=0$;

(b) $v_{n}$ is positive, continuous and concave on $[0,+\infty)$; and

(c) $v_{n}$ is differentiable on $(0,+\infty)$ with $v_{n}^{\prime}(t)>0$ for each $t>0$.

Now let $u$ be a rational separable utility function. The components of the lower and upper gradient sequences $\underline{v}^{\prime}=\left(\underline{v}_{1}^{\prime}, \underline{v}_{2}^{\prime}, \ldots\right)$ and $\bar{v}^{\prime}=\left(\bar{v}_{1}^{\prime}, \bar{v}_{2}^{\prime}, \ldots\right)$ are given by

$$
\underline{v}_{n}^{\prime}=\lim _{t \rightarrow+\infty} v_{n}^{\prime}(t) \quad \text { and } \quad \bar{v}_{n}^{\prime}=\lim _{t \rightarrow 0} v_{n}^{\prime}(t) .
$$

Following Aliprantis [1, Theorem 6.7], we have the following result.

Proposition 4.1. Let $u: \ell_{+}^{p} \rightarrow \mathbb{R}$ be rational utility function given by $u(x)=\sum_{n} v_{n}\left(x_{n}\right)$. If the preference relations represented by $u$ are $\omega$-uniformly proper for some $\omega \in \ell^{p}$ strictly positive, then

(a) the lower gradient $\underline{v}^{\prime}$ is non-zero and belongs to $\ell_{+}^{q}$; and

(b) there exists some $k \in \mathbb{N}$ such that

$$
\left(0,0, \ldots, 0, \bar{v}_{k}^{\prime}, \bar{v}_{k+1}^{\prime}, \ldots\right) \in \ell_{+}^{q} .
$$

It follows that if $\mathcal{E}$ is an economy with rational separable utility function such that $\mathcal{E}$ is $\omega$ uniformly proper and $\omega$ is strictly positive, then $\mathcal{E}$ is separably proper. We provide hereafter an example of a rational separable utility function which is separably proper but which is not uniformly proper.

Example 4.5. Consider the rational separable utility function $u: \ell_{+}^{p} \rightarrow \mathbb{R}$ defined by

$$
v_{0}(t)=t \quad \text { and } \quad \forall n \geqslant 1, \quad v_{n}(t)=\frac{1-\exp \left(-t n^{2}\right)}{n^{2}} .
$$

For each $n \geqslant 1, v_{n}^{\prime}(t)=\exp \left(-n^{2} t\right)$. It follows that

$$
\underline{v}^{\prime}=(1,0,0, \ldots) \quad \text { and } \quad \bar{v}^{\prime}=(1,1,1, \ldots) .
$$

It follows that $u$ is not uniformly proper. However if $e=\left(e_{n}\right)_{n}$ is defined by $e_{0}=1$ and for each $n \geqslant 1$ by $e_{n}=1 / n$ then

$$
v^{\prime}(e)=\left(e^{-n}\right)_{n} \in \ell_{+}^{p}
$$

Hence $u$ is separably proper.

\section{Proof of Theorem 3.3 and Theorem 3.9}

Since $E$ is a separable and reflexive Banach space, it follows that $E^{*}$ is norm-separable. Let $\left(p_{i}\right)_{i \in \mathbb{N}}$ be a norm-dense sequence in the closed unit ball of $E^{*}$ and define for each $x, y$ in $E$,

$$
d(x, y)=\sum_{i \in \mathbb{N}} \frac{\left|\left\langle p_{i}, x-y\right\rangle\right|}{2^{i}} .
$$

The topology defined by this distance coincide with the $w$-topology on norm-bounded subsets of $E$. Moreover, the $d$-topology is separable and the Borel $\sigma$-algebra generated by $d$ coincide with the Borel $\sigma$-algebra $\mathcal{B}$ generated by the norm-topology and the $w$-topology. 
Let $\mathcal{E}$ be an economy satisfying Assumptions 3.1-3.4. Suppose that $\mathcal{E}$ is either strong-uniformly proper or separably proper. The correspondence $X$ is graph measurable. Applying Theorem B.1, there exists a sequence $\left(f_{k}\right)_{k}$ of measurable selections of $X$ such that for each $a \in \Omega, X(a)=$ $s-\operatorname{cl}\left\{f_{k}(a): k \in \mathbb{N}\right\}$. For every $k \in \mathbb{N}$, we let $R_{k}$ be the correspondence from $\Omega$ into $E$, defined by $R_{k}(a)=\left\{x \in E_{+}: x \succeq_{a} f_{k}(a)\right\}$. For each $\nu \in \mathbb{N}$, we let $X_{\nu}: a \mapsto X_{\nu}(a):=X(a) \cap \nu B$ and $R_{k, \nu}: a \rightarrow R_{k, \nu}(a):=R_{k}(a) \cap \nu B$, where $B$ is the closed unit ball in $E$.

Claim 5.1. There exists ${ }^{10}$ a sequence $\left(\sigma^{n}\right)_{n}$ of measurable partitions $\sigma^{n}=\left(A_{i}^{n}\right)_{i \in S^{n}}$ of $(\Omega, \mathcal{A})$, and a sequence $\left(A^{n}\right)_{n}$ of finite sets $A^{n}=\left\{a_{i}^{n}: i \in S^{n}\right\}$ subordinated to the measurable partition $\sigma^{n}$, satisfying ${ }^{11}$ for each $a \in \Omega$,

(i)

$$
\lim _{n}\left\|e^{n}(a)-e(a)\right\|=0 \quad \text { and } \quad \forall k \in \mathbb{N}, \quad \lim _{n}\left\|f_{k}^{n}(a)-f_{k}(a)\right\|=0 ;
$$

(ii) for each $\nu \in \mathbb{N}$, for each sequence $\left(x^{n}\right)_{n}$ of $E$, d-converging to $x \in E$ and for every $k \in \mathbb{N}$,

$$
\lim _{n} d\left(x^{n}, R_{k, \nu}^{n}(a)\right)=d\left(x, R_{k, \nu}(a)\right) ;
$$

(iii) if we pose $e^{12} g(a):=\rho[e(a)]$ then $g$ is an integrable function satisfying

$$
\forall n \in \mathbb{N}, \quad \rho\left[e^{n}(a)\right] \leqslant 1+g(a) .
$$

Proof. If $f$ is a function from $\Omega$ to $E$, then we let $\{f()$.$\} be the correspondence from \Omega$ into $E$ defined for each $a \in \Omega$, by $\{f()\}.(a):=\{f(a)\}$. Note that if $f$ is measurable then $f$ is Bochner integrable if and only if $\|f()\|:. a \mapsto\|f(a)\|$ from $\Omega$ to $\mathbb{R}_{+}$is integrable.

Let $Z:=E \times E$ and consider the following distance $\delta$ on $Z$ defined for each $x=\left(x_{1}, x_{2}\right)$ and $y=\left(y_{1}, y_{2}\right)$ in $Z$ by

$$
\delta(x, y):=\left\|x_{1}-y_{1}\right\|+d\left(x_{2}, y_{2}\right) .
$$

The metric space $(Z, \delta)$ is complete and separable. Let $\mathcal{G}:=\{\rho[e()]$.$\} and ^{13}$

$$
\mathcal{F}:=\left\{\{e(.)\} \times\{0\},\left\{f_{k}(.)\right\} \times\{0\},\{0\} \times R_{k}: k \in \mathbb{N}\right\} .
$$

Now apply Theorem B.6. let

We construct a sequence $\left(\mathcal{E}^{n}\right)_{n}$ of economies with finitely many consumers converging to $\mathcal{E}$. We

$$
\mathcal{E}^{n}:=\left(\left(I^{n}, 2^{I^{n}}, \sigma\right), E, X^{n}, \succ^{n}, e^{n}\right)
$$

where $I^{n}=\left\{i \in S^{n}: \mu\left(A_{i}^{n}\right) \neq 0\right\}$ is the finite set of consumers; $\sigma$ is the counting measure on $I^{n}$; for each agent $i \in I^{n}$, the consumption set is defined by $X_{i}^{n}:=\mu\left(A_{i}^{n}\right) X\left(a_{i}^{n}\right)=E_{+}$, the initial endowment is defined by $e_{i}^{n}:=\mu\left(A_{i}^{n}\right) e\left(a_{i}^{n}\right)$ and the preference relation is defined by $x^{\prime} \succ_{i}^{n} x$ if and only if $\left(x^{\prime} / \mu\left(A_{i}^{n}\right)\right) \succ_{a_{i}^{n}}\left(x / \mu\left(A_{i}^{n}\right)\right)$. In particular, the correspondence of strictly preferred bundles $P_{i}^{n}$ from $X_{i}^{n}$ to $X_{i}^{n}$ is defined by $P_{i}^{n}(x)=\mu\left(A_{i}^{n}\right) P_{a_{i}^{n}}\left(x / \mu\left(A_{i}^{n}\right)\right)$, for each $x \in X_{i}^{n}$.

Claim 5.2. There exists a feasible allocation $\left(x_{i}^{n}\right)_{i \in I^{n}}$ for the finite economy $\mathcal{E}^{n}$, a non-zero price $p^{n}$ and $a w^{*}$-compact set $K \subset E^{*}$ such that

\footnotetext{
${ }^{10}$ We refer to Appendix B for definitions and notations.

${ }^{11}$ Following notations of Section B.2, if $f$ is function from $\Omega$ to $E$, then for each $n,\{f(.)\}^{n}=\left\{f^{n}().\right\}$.

12 The functional $\rho$ is given by Assumption 3.4.

${ }^{13}$ If $F$ and $G$ are two correspondences from $\Omega$ to $E$, then we let $F \times G$ be the correspondence from $\Omega$ to $E \times E$, defined for each $a \in \Omega$ by $(F \times G)(a)=F(a) \times G(a)$.
} 
(1) for each $i \in I^{n},\left\langle p^{n}, x_{i}^{n}\right\rangle=\left\langle p^{n}, e_{i}^{n}\right\rangle$, and $z \in P_{i}^{n}\left(x_{i}^{n}\right)$ implies $\left\langle p^{n}, z\right\rangle \geqslant\left\langle p^{n}, x_{i}^{n}\right\rangle$; and

(2) $p^{n} \in K$ with $\left\langle p^{n}, \omega\right\rangle=1$.

Proof. If the economy $\mathcal{E}$ is strong-uniformly proper then each economy $\mathcal{E}^{n}$ satisfies the assumptions of Theorem A.1. In particular if we let $K:=\left\{q \in E^{*}:\langle q, \omega\rangle=1\right.$ and $\left.\langle q, \Gamma\rangle>0\right\}$, where $\left.\Gamma=\left\{x \in E:\left\langle\alpha, x^{+}\right\rangle\right\rangle\left\langle\beta, x^{-}\right\rangle\right\}$, then Claim 5.2 is proved.

If the economy $\mathcal{E}$ is separably proper then for each $n$ large enough, $\alpha_{k} \omega_{k}^{n}>0$. Hence the economy $\mathcal{E}^{n}$ satisfies the assumptions of Theorem A.3. In particular for each $n,\left\|p^{n}\right\|_{q} \leqslant\left(1 / \alpha_{k} \omega_{k}^{n}\right)\|\beta\|_{q}$. Since $\left(\omega_{k}^{n}\right)_{n}$ is norm-convergent to $\omega^{k}$, it follows that the sequence $\left(p^{n}\right)_{n}$ lies in a norm-bounded set $K \subset \ell^{q}$. In particular, Claim 5.2 is proved.

If we denote by $x^{n}$ and $e^{n}$ the Bochner integrable mappings defined by

$$
x^{n}:=\sum_{i \in I^{n}} x_{i}^{n} \chi_{A_{i}^{n}} \quad \text { and } \quad e^{n}:=\sum_{i \in I^{n}} e_{i}^{n} \chi_{A_{i}^{n}}
$$

then

$$
\int_{\Omega} x^{n} d \mu=\int_{\Omega} e^{n} d \mu
$$

$$
\begin{gathered}
\text { for a.e. } a \in \Omega, \quad\left\langle p^{n}, x^{n}(a)\right\rangle=\left\langle p^{n}, e^{n}(a)\right\rangle \quad \text { and } \quad z \in P_{a}^{n}\left(x^{n}(a)\right) \Rightarrow\left\langle p^{n}, z\right\rangle \geqslant\left\langle p^{n}, e^{n}(a)\right\rangle \\
p^{n} \in K \quad \text { and } \quad\left\langle p^{n}, \omega\right\rangle=1 .
\end{gathered}
$$

The set $K$ is $w^{*}$-compact. Since $E$ is norm-separable, passing to a subsequence if necessary, we can suppose that $\left(p^{n}\right)_{n} w^{*}$-converge to a non-zero price $p \in E^{*}$ which satisfies $\langle p, \omega\rangle=1$.

Now we want to apply a Fatou's Lemma to the sequence $\left(x^{n}\right)$. It is only at this step that we need Assumption 3.4. For each $z \in E_{+},\|z\| \leqslant \rho(z)$; and for each $y \in E_{+}, \rho(z+y)=\rho(z)+\rho(y)$. Hence from (5.1), we have

$$
\int_{\Omega}\left\|x^{n}(a)\right\| d \mu(a) \leqslant \int_{\Omega} \rho\left[x^{n}(a)\right] d \mu(a)=\int_{\Omega} \rho\left[e^{n}(a)\right] d \mu(a) .
$$

Applying Claim 5.1, the sequence of Bochner integrable mappings $\left(x^{n}\right)_{n}$ is mean norm-bounded, i.e.

$$
\sup _{n} \int_{\Omega}\left\|x^{n}(a)\right\| d \mu(a)<+\infty .
$$

Passing to a subsequence if necessary, we can suppose that $w-\lim _{n} \int_{\Omega} x^{n} d \mu$ exists in $E$. Applying Fatou's Lemma (Theorem C.1) of Cornet-Martins-da-Rocha [10], there exists a Bochner integrable mapping $x$ from $\Omega$ to $E$ such that

$$
\begin{gathered}
\int_{\Omega} x d \mu \leqslant w-\lim _{n} \int_{\Omega} x^{n} d \mu \\
x(a) \in \overline{\mathrm{co}} w-\operatorname{ls}_{n}\left\{x_{n}(a)\right\} \quad \text { a.e. }
\end{gathered}
$$

Claim 5.3. The Bochner integrable mapping $x$ satisfies $\int_{\Omega} x d \mu \leqslant \int_{\Omega} e d \mu$.

Proof. This is a direct consequence of Claim 5.1, (5.1) and (5.4).

Claim 5.4. For almost every $a \in \Omega, x(a) \in E_{+}$and $z \in P_{a}(x(a))$ implies $\langle p, z\rangle \geqslant\langle p, e(a)\rangle$. 
Proof. Consider $\Omega_{0}=\bigcup_{n \in \mathbb{N}} \Omega \backslash\left(\cup_{i \in I^{n}} A_{i}^{n}\right)$, then $\mu\left(\Omega_{0}\right)=0$. Let $\Omega^{\prime}$ be a measurable subset of $\Omega \backslash \Omega_{0}$ with $\mu\left(\Omega \backslash \Omega^{\prime}\right)=0$ and such that all almost everywhere assumptions and properties are satisfied for each $a \in \Omega^{\prime}$.

Since $X(a)=E_{+}$is closed convex, we have that for each $a \in \Omega^{\prime}, x(a) \in X(a)$. We will now prove that for each $a \in \Omega^{\prime}$, if $z \in P_{a}(x(a))$ then $\langle p, z\rangle \geqslant\langle p, e(a)\rangle$. Let $a \in \Omega^{\prime}$ and let $z \in P_{a}(x(a))$. Since $E_{+}=s-\operatorname{cl}\left\{f_{k}(a): k \in \mathbb{N}\right\}$, we can suppose (extracting a subsequence if necessary) that $\left(f_{k}(a)\right)_{k}$ is norm-convergent to $z$. But $P_{a}(x(a))$ is norm-open in $E_{+}$, thus there exists $k_{0} \in \mathbb{N}$, such that for each $k \geqslant k_{0}, f_{k}(a) \in P_{a}(x(a))$. To prove that $\langle p, z\rangle \geqslant\langle p, e(a)\rangle$, it is sufficient to prove that for each $k$ large enough, $\left\langle p, f_{k}(a)\right\rangle \geqslant\langle p, e(a)\rangle$. Now, let $k \geqslant k_{0}$.

Claim 5.5. There exists an increasing function $\varphi: \mathbb{N} \rightarrow \mathbb{N}$ such that

$$
\forall n \in \mathbb{N}, \quad f_{k}^{\varphi(n)}(a) \in P_{a}^{\varphi(n)}\left(x^{\varphi(n)}(a)\right) .
$$

Proof. Suppose that for each increasing function $\varphi: \mathbb{N} \rightarrow \mathbb{N}$, there exists an increasing function $\phi: \mathbb{N} \rightarrow \mathbb{N}$, such that:

$$
\forall n \in \mathbb{N}, \quad x^{\varphi \circ \phi(n)}(a) \in R_{k}^{\varphi \circ \phi(n)}(a) .
$$

Let $\ell \in w-\operatorname{ls}\left\{x^{n}(a): n \in \mathbb{N}\right\}$, then there exists a subsequence $\left(x^{\varphi(n)}(a)\right)_{n} w$-converging to $\ell$. In particular $\left(x^{\varphi \circ \phi(n)}(a)\right)_{n}$ is $w$-convergent to $\ell$. It follows that there exists $\nu>0$ such that for each $n, x^{\varphi \circ \phi(n)}(a)$ belongs to $\nu B$. In particular, $d\left(x^{\varphi \circ \phi(n)}(a), R_{k, \nu}^{\varphi \circ \phi}(a)\right)=0$. Applying Claim 5.1, it follows that $d\left(\ell, R_{k, \nu}(a)\right)=0$. Since $R_{k, \nu}(a)$ is $w$-closed and $d$ coincide with $w$ on $\nu B$, we have that $\ell \in R_{k}(a)$. Thus $w-\operatorname{ls}\left\{x^{n}(a)\right\} \subset R_{k}(a)$, and under Assumption 3.2, this implies that $\overline{\mathrm{co}} w-\operatorname{ls}_{n}\left\{x_{n}(a)\right\} \subset R_{k}(a)$. It follows that $x(a) \in R_{k}(a)$, i.e. $f_{k}(a) \notin P_{a}(x(a))$ : contradiction.

With Claim 5.5 and (5.2), for each $n,\left\langle p^{\varphi(n)}, f_{k}^{\varphi(n)}(a)\right\rangle \geqslant\left\langle p^{\varphi(n)}, e^{\varphi(n)}(a)\right\rangle$. Passing to the limit, we get that $\left\langle p, f_{k}(a)\right\rangle \geqslant\langle p, e(a)\rangle$.

Now let $\bar{x}$ be the Bochner integrable mapping from $\Omega$ to $\ell^{p}$ defined by

$$
\forall a \in \Omega, \quad \bar{x}(a)=x(a)+(1 / \mu(\Omega)) \int_{\Omega}(e-x) d \mu .
$$

Claim 5.6. The pair $(\bar{x}, p)$ is an equilibrium of $\mathcal{E}$.

Proof. Since $\int_{\Omega}(e-x) d \mu \geqslant 0$, Assumption 3.1 implies that $\bar{x}(a) \in E_{+}$and $P_{a}(\bar{x}(a)) \subset P_{a}(x(a))$. In particular the allocation $\bar{x}$ is feasible and for each $a \in \Omega^{\prime}$, if $z \in P_{a}(\bar{x}(a))$ then $\langle p, z\rangle \geqslant\langle p, e(a)\rangle$. Since $\succ_{a}$ is monotone, it follows that $\bar{x}(a)$ belongs to the norm-closure of $P_{a}(\bar{x}(a))$, in particular $\langle p, \bar{x}(a)\rangle \geqslant\langle p, e(a)\rangle$. But $\int_{\Omega} \bar{x} d \mu=\int_{\Omega} e d \mu$, it follows that for almost every $a \in \Omega,\langle p, \bar{x}(a)\rangle=$ $\langle p, e(a)\rangle$. To prove that $(\bar{x}, p)$ is an equilibrium, it is now sufficient to prove that for almost every $a \in \Omega$,

$$
\inf \left\{\langle p, z\rangle: z \in E_{+}\right\}<\langle p, e(a)\rangle \text {. }
$$

Let $B:=\left\{a \in \Omega^{\prime}:\langle p, e(a)\rangle>0\right\}$. The set $B$ is measurable and since $\langle p, \omega\rangle=1, \mu(B) \neq 0$. Now for each $a \in B, \inf \left\{\langle p, z\rangle: z \in E_{+}\right\}<\langle p, e(a)\rangle$ and $z \in P_{a}(\bar{x}(a)) \Rightarrow\langle p, z\rangle \geqslant\langle p, \bar{x}(a)\rangle$. It follows that

$$
\forall a \in B, \quad z \in P_{a}(\bar{x}(a)) \Rightarrow\langle p, z\rangle>\langle p, \bar{x}(a)\rangle
$$


The preference relation $\succ_{a}$ is monotone, i.e. for each $z>0, \bar{x}(a)+z \in P_{a}(\bar{x}(a))$. It follows that for each $z>0,\langle p, z\rangle>0$. Now from Assumption 3.1, for each $a \in \Omega, e(a)>0$, hence

$$
\inf \left\{\langle p, z\rangle: z \in E_{+}\right\}=0<\langle p, e(a)\rangle .
$$

\section{Appendix A. Finitely many CONSUmers}

We suppose in this section that the economy is finite in the sense that the set of consumers $(\Omega, \mathcal{A}, \mu)$ is $\left(I, 2^{I}, \sigma\right)$ where $I$ is a finite set, $2^{I}$ is the $\sigma$-algebra of all subsets of $I$ and $\sigma$ is the counting measure.

A.1. The general case. If $\mathcal{E}$ is strong-uniformly proper then we denote by $\Gamma$ the norm-open convex cone defined by $\left.\Gamma=\left\{x \in E:\left\langle\alpha, x^{+}\right\rangle\right\rangle\left\langle\beta, x^{-}\right\rangle\right\}$.

Theorem A.1. Let $\mathcal{E}$ be a finite economy satisfying Assumptions 3.1-3.4. If $\mathcal{E}$ is strong-uniformly proper then there exists a pair $(x, p)$ consisting of a feasible allocation $x$ and a non-zero price $p$ such that

(1) for each $i \in I,\left\langle p, x_{i}\right\rangle=\left\langle p, e_{i}\right\rangle$, and $z \in P_{i}\left(x_{i}\right)$ implies $\langle p, z\rangle \geqslant\left\langle p, x_{i}\right\rangle$;

(2) $\langle p, \omega\rangle=1$ and $\langle p, \Gamma\rangle>0$.

Proof. Since order intervals $[0, x]=\{y \in E: 0 \leqslant y \leqslant x\}$ are $w$-compact, ${ }^{14}$ following Florenzano [13], there exists a feasible allocation $x=\left(x_{i}\right)_{i}$ such that ${ }^{15}$

$$
0 \notin G(x):=\operatorname{co} \bigcup_{i \in I}\left[P_{i}\left(x_{i}\right)-e_{i}\right]
$$

Lemma A.2. $G(x) \cap-\Gamma=\emptyset$.

Proof. To see this, ${ }^{16}$ assume by way of contradiction that $G(x) \cap-\Gamma \neq \emptyset$. Then there exist $\gamma \in \Gamma$, $\left(\lambda_{i}\right)_{i}$ with $\lambda_{i} \geqslant 0, \sum_{i} \lambda_{i}=1$ and $\left(z_{i}\right)_{i}$ with $z_{i} \in P_{i}\left(x_{i}\right)$ such that

$$
\sum_{i} \lambda_{i} z_{i}+\gamma=\sum_{i} \lambda_{i} e_{i}
$$

Suppose first that $\gamma \geqslant 0$. For each $i \in I$, we set $y_{i}:=z_{i}+\gamma$. Then $y_{i} \succ_{i} z_{i}$ for each $i$ since preference relations are strictly monotone, whence $y_{i} \succ_{i} x_{i}$ by transitivity. One the other hand,

$$
\sum_{i} \lambda_{i} y_{i}=\sum_{i} \lambda_{i} e_{i}
$$

and we have thus got a contradiction.

Thus suppose that $\gamma^{-} \neq 0$. We must have $\gamma^{-} \leqslant \sum_{i} \lambda_{i} z_{i}$, so by the Riesz decomposition theorem there exist elements $u_{i} \geqslant 0$ such that $u_{i} \leqslant z_{i}$ and $\sum_{i} \lambda_{i} u_{i}=\gamma^{-}$. Set for each $i$,

$$
v_{i}=\frac{\left\langle\beta, u_{i}\right\rangle}{\left\langle\beta, \gamma^{-}\right\rangle} \gamma^{+}
$$

\footnotetext{
${ }^{14}$ Since $E$ is a Banach lattice, the order interval $[0, x]$ is a subset of $\|x\| B$. Since $E$ is separable and reflexive then $B$ is $w$-compact.

${ }^{15}$ In fact $x$ is an Edgeworth equilibrium of $\mathcal{E}$.

${ }^{16}$ The argument given in the sequel to establish this lemma is taken from Zame [30] and Podczeck [25].
} 
Since $\left\langle\alpha, \gamma^{+}\right\rangle>\left\langle\beta, \gamma^{-}\right\rangle$by definition of $\Gamma$,

$$
\left\langle\alpha, v_{i}\right\rangle=\frac{\left\langle\beta, u_{i}\right\rangle}{\left\langle\beta, \gamma^{-}\right\rangle}\left\langle\alpha, \gamma^{+}\right\rangle \geqslant\left\langle\beta, u_{i}\right\rangle,
$$

with strict inequality if $u_{i} \neq 0$. Hence because $u_{i} \leqslant z_{i}$ and $v_{i} \geqslant 0$, we have $z_{i}-u_{i}+v_{i} \succeq_{i} z_{i}$ for each $i$ (in fact, $z_{i}-u_{i}+v_{i} \succ_{i} z_{i}$ in case $u_{i} \neq 0$ ), and therefore by transitivity of preference relations, $z_{i}-u_{i}+v_{i} \succ_{i} x_{i}$. Also

$$
\sum_{i} \lambda_{i}\left(z_{i}-u_{i}+v_{i}\right)=\sum_{i} \lambda_{i} z_{i}-\gamma^{-}+\frac{\left\langle\beta, \sum_{i} \lambda_{i} u_{i}\right\rangle}{\left\langle\beta, \gamma^{-}\right\rangle} \gamma^{+}=\sum_{i} e_{i},
$$

again we get a contradiction.

Following Lemma A.2, since $\Gamma$ is norm-open, it follows from the separation theorem that there exists some non-zero linear functional $p \in E^{*}$ with $\langle p, g\rangle \geqslant-\langle p, \gamma\rangle$ for each $g \in G(x)$ and $\gamma \in \Gamma$. It is now routine to prove that $(x, p)$ satisfies properties (1) and (2) of Theorem A.1.

A.2. The separable case. We recall that a utility function $u: \ell_{+}^{p} \rightarrow \mathbb{R}$ is called separable if there exists for each $n$, a function $v_{n}:[0,+\infty) \rightarrow \mathbb{R}$ concave and strictly increasing such that

$$
\forall x \in \ell_{+}^{p}, \quad u(x)=\sum_{n \in \mathbb{N}} v_{n}\left(x_{n}\right) .
$$

Theorem A.3. Let $\mathcal{E}$ be a finite economy satisfying Assumptions 3.1-3.4. If $\mathcal{E}$ is separably proper then there exists a pair $(x, p)$ consisting of a feasible allocation $x$ and a non-zero price $p$ such that

(1) for each $i \in I,\left\langle p, x_{i}\right\rangle=\left\langle p, e_{i}\right\rangle$, and $z \in P_{i}\left(x_{i}\right)$ implies $\langle p, z\rangle \geqslant\left\langle p, x_{i}\right\rangle$;

(2) $\langle p, \omega\rangle=1$ and $\|p\|_{q} \leqslant\left(1 / \alpha_{k} \omega_{k}\right)\|\beta\|_{q}$.

The proof of Theorem A.3 is mostly inspired by the proof of Theorem 3 in Araujo-Monteiro [4].

Proof. We prove Theorem A.3 in two steps. For the first step, we suppose that the economy satisfies an additional assumption on the initial endowments.

Step 1: Strictly positive initial endowments. Suppose that for each $i, e_{i}$ is strictly positive. Let $E^{\omega}$ be the vector space of all $z \in \ell^{p}$ such that there exists $r>0$ satisfying $-r \omega \leqslant z \leqslant r \omega$. From Lemma 1 in Araujo-Monteiro [4], there exists a pair $(x, p)$ consisting of a feasible allocation $^{17} x$ and a non-zero linear functional $p: E^{\omega} \rightarrow \mathbb{R}$ such that $p$ is positive, i.e. $\langle p, z\rangle \geqslant 0$ for each $z \in E_{+}^{\omega}$; $\langle p, \omega\rangle=1$ and such that

$$
\forall i \in I, \quad\left\langle p, x_{i}\right\rangle=\left\langle p, e_{i}\right\rangle \quad \text { and } \quad z \in P_{i}\left(x_{i}\right) \cap E^{\omega} \Rightarrow\langle p, z\rangle \geqslant\left\langle p, e_{i}\right\rangle .
$$

Now there exists $i$ with $\left\langle p, e_{i}\right\rangle>0$, and since $u_{i}$ is strictly monotone, $p$ is strictly positive, i.e. $\langle p, z\rangle>0$ for each $0 \neq z \in E_{+}^{\omega}$. In particular $\left\langle p, e_{i}\right\rangle>0$ for each $i \in I$. By the concave alternative (see Lemma 5 in [4]), for each $i$ there exists $\lambda_{i}>0$ such that

$$
\forall z \in E_{+}^{\omega}, \quad u_{i}(z)-u_{i}\left(x_{i}\right) \leqslant \lambda_{i}\left\langle p, z-x_{i}\right\rangle .
$$

For $z \in \ell_{+}^{p}$ we define $S(z)=\left\{h \in \ell^{p}: \exists t>0, z+t h \geqslant 0\right\}$ and $I(z)=S(z) \cap-S(z)$. Using (A.1) like in [4], we have

$$
\forall h \in I\left(x_{i}\right) \cap E_{+}^{\omega}, \quad \lambda_{i}\langle p, h\rangle \leqslant \sum_{n} v_{i, n}^{-}\left(x_{i, n}\right) h_{n} .
$$

\footnotetext{
${ }^{17}$ Note that if $x$ is a feasible allocation then $x_{i} \in E^{\omega}$.
} 
Since $e_{i}$ is strictly positive for each $i$, we have that $b:=\inf \left\{e_{i}: i \in I\right\}$ is strictly positive. Hence $E^{b}$ is norm-dense in $\ell^{p}$. From this we conclude that if $p$ is norm-continuous on $E^{b}$ then we can extend it to a linear functional still noted $p$ in $\ell^{q}$, such that $(x, p)$ satisfies

$$
\forall i \in I, \quad\left\langle p, x_{i}\right\rangle=\left\langle p, e_{i}\right\rangle \quad \text { and } \quad z \in P_{i}\left(x_{i}\right) \Rightarrow\langle p, z\rangle \geqslant\left\langle p, e_{i}\right\rangle .
$$

So let us prove that $p$ is norm-continuous on $E^{b}$.

We define for each $n, I_{n}:=\left\{i \in I: x_{i, n} \geqslant e_{i, n}\right\}$ and for each $i$ we define $N_{i}=\{n \in \mathbb{N}: i=$ $\min I_{n}$ \}. Since $x$ is a feasible allocation, we have $I_{n} \neq \emptyset$ for every $n$ and $\left(N_{i}\right)_{i}$ is a partition of $\mathbb{N}$. Take $h \in E^{b}$, there exists $r>0$ such that $-r e_{i} \leqslant h \leqslant r e_{i}$ for each $i$. Now let $h^{i} \in E^{b}$ be defined by

$$
h^{i}=\left(h_{n}^{i}\right)_{n} \quad \text { where } \quad h_{n}^{i}=\left\{\begin{array}{ccc}
\left|h_{n}\right| & \text { if } & n \in N_{i} \\
0 & \text { if } & n \notin N_{i}
\end{array}\right.
$$

As $h^{i}$ belongs to $I\left(x_{i}\right)$, it follows from (A.2) that ${ }^{18}$

$$
\lambda_{i}\left\langle p, h^{i}\right\rangle \leqslant \sum_{n \in N_{i}} v_{i, n}^{-}\left(x_{i, n}\right)\left|h_{n}\right| \leqslant \sum_{n \in N_{i}} v_{i, n}^{-}\left(e_{i, n}\right)\left|h_{n}\right| .
$$

Since $p$ is positive, we have that $|\langle p, h\rangle| \leqslant \sum_{i}\left\langle p, h^{i}\right\rangle$. It follows that

$$
|\langle p, h\rangle| \leqslant \sum_{i}\left(1 / \lambda_{i}\right) \sum_{n \in N_{i}} v_{i, n}^{-}\left(e_{i, n}\right)\left|h_{n}\right| .
$$

We define $u_{i}^{\prime}(z) \cdot h=\lim _{r \rightarrow 0}(1 / r)\left(u_{i}(z+r h)-u_{i}(z)\right)$ for each $z \in \ell_{+}^{p}$ and each $h \in S(z)$. It follows from (A.1) and separable properness that

$$
0<\alpha_{k} \omega_{k}<u_{i}^{\prime}\left(x_{i}\right) \cdot \omega \leqslant \lambda_{i} .
$$

In particular

$$
|\langle p, h\rangle| \leqslant\left(1 / \alpha_{k} \omega_{k}\right) \sum_{i} \sum_{n \in N_{i}} v_{i, n}^{-}\left(e_{i, n}\right)\left|h_{n}\right| \leqslant\left(1 / \alpha_{k} \omega_{k}\right) \sum_{n \in \mathbb{N}} \beta_{n}\left|h_{n}\right| \leqslant\left(1 / \alpha_{k} \omega_{k}\right)\|\beta\|_{q}\|h\|_{p} .
$$

From separable properness we have that $\|\beta\|_{q}<+\infty$. This proves the norm-continuity of $p$ on $E^{b}$.

Step 2: Positive initial endowments. Let $\mathcal{E}$ be a separably proper finite economy satisfying Assumptions 3.1-3.4. Let $v$ be a strictly positive vector of $\ell^{p}$ and consider $\mathcal{E}^{n}$ the economy defined by $\mathcal{E}=\left(I, \ell^{p}, X, \succ, e^{n}\right)$ where $e_{i}^{n}:=e_{i}+(1 / n) v$. Since $v_{i}^{-}\left(e_{i}^{n}\right) \leqslant v_{i}^{-}\left(e_{i}\right)$, the economy $\mathcal{E}^{n}$ is separably proper and satisfies Assumptions 3.1-3.4. Applying Step 1, there exists a pair $\left(x^{n}, p^{n}\right)$ consisting of a feasible allocation $x^{n}$ and a non-zero price $p^{n}$ such that for each $i \in I$, $\left\langle p, x_{i}^{n}\right\rangle=\left\langle p, e_{i}^{n}\right\rangle, z \in P_{i}\left(x_{i}^{n}\right)$ implies $\left\langle p^{n}, z\right\rangle \geqslant\left\langle p^{n}, x_{i}^{n}\right\rangle,\left\langle p^{n}, \omega^{n}\right\rangle=1$ and $\left\|p^{n}\right\|_{q} \leqslant\left(1 / \alpha_{k} \omega_{k}^{n}\right)\|\beta\|_{q}$.

Since the sequence $\left(\omega_{k}^{n}\right)_{n}$ is norm-convergent to $\omega_{k}$, it follows that the sequence $\left(p^{n}\right)_{n}$ is normbounded, and passing to a subsequence if necessary, we can suppose that the sequence $\left(p^{n}\right)_{n}$ is $w^{*}$-convergent to a price $p \in \ell^{q}$ with $\|p\|_{q} \leqslant\left(1 / \alpha_{k} \omega_{k}\right)\|\beta\|_{q}$. Moreover, since $\left\langle p^{n}, \omega^{n}\right\rangle=1$ it follows that $\langle p, \omega\rangle=1$.

For each $i, x_{i}^{n}$ belongs to the interval $[0, \omega+v]$, in particular, passing to a subsequence if necessary, we can suppose that $\left(x_{i}^{n}\right)_{n}$ is $w$-convergent to $x_{i} \in \ell_{+}^{p}$. Moreover, since $\sum_{i} x_{i}^{n}=\omega^{n}$, we have that $x$ is a feasible allocation for the economy $\mathcal{E}$. It is now routine to prove that for each $i \in I,\left\langle p, x_{i}\right\rangle=\left\langle p, e_{i}\right\rangle$, and $z \in P_{i}\left(x_{i}\right)$ implies $\langle p, z\rangle \geqslant\left\langle p, x_{i}\right\rangle$.

\footnotetext{
${ }^{18}$ Note that $v_{i, n}^{-}$is a decreasing function.
} 


\section{Appendix B. Measurable CORRespondences}

We consider $(\Omega, \mathcal{A}, \mu)$ a finite complete measure space and $(D, d)$ a separable metric space. We recall that a function $f: \Omega \rightarrow D$ is measurable if for each open set $G \subset D, f^{-1}(G) \in \mathcal{A}$ where $f^{-1}(G):=\{a \in \Omega: f(a) \in G\}$. A correspondence $F: \Omega \rightarrow D$ is graph measurable if $G_{F}:=\{(a, x) \in \Omega \times D: x \in F(a)\} \in \mathcal{A} \otimes \mathcal{B}(D)$, where $\mathcal{B}(D)$ is the $\sigma$-algebra of Borelian subsets of $D$.

B.1. Measurable selections. Following Aumann [8], graph measurable correspondences have measurable selections.

Theorem B.1. Consider $F$ a graph measurable correspondence from $\Omega$ into $D$ with non-empty values. If $(D, d)$ is complete then there exists a sequence $\left(z_{n}\right)_{n}$ of measurable selections of $F$, such that for each $a \in \Omega,\left(z_{n}(a)\right)_{n}$ is d-dense in $F(a)$.

\section{B.2. Discretization of measurable correspondences.}

Definition B.2. A partition $\sigma=\left(A_{i}\right)_{i \in I}$ of $\Omega$ is a measurable partition if for each $i \in I$, the set $A_{i}$ is non-empty and belongs to $\mathcal{A}$. A finite subset $A^{\sigma}$ of $\Omega$ is subordinated to the partition $\sigma$ if there exists a family $\left(a_{i}\right)_{i \in I} \in \prod_{i \in I} A_{i}$ such that $A^{\sigma}=\left\{a_{i}: i \in I\right\}$.

Given a couple $\left(\sigma, A^{\sigma}\right)$ where $\sigma=\left(A_{i}\right)_{i \in I}$ is a measurable partition of $\Omega$, and $A^{\sigma}=\left\{a_{i}: i \in I\right\}$ is a finite set subordinated to $\sigma$, we consider $\phi\left(\sigma, A^{\sigma}\right)$ the application which maps each measurable function $f$ to a simple measurable function $\phi\left(\sigma, A^{\sigma}\right)(f)$, defined by

$$
\phi\left(\sigma, A^{\sigma}\right)(f):=\sum_{i \in I} f\left(a_{i}\right) \chi_{A_{i}},
$$

where $\chi_{A_{i}}$ is the characteristic ${ }^{19}$ function associated to $A_{i}$.

Definition B.3. A function $s: \Omega \rightarrow D$ is called a simple function subordinated to $f$ if there exists a couple $\left(\sigma, A^{\sigma}\right)$ where $\sigma$ is a measurable partition of $\Omega$, and $A^{\sigma}$ is a finite set subordinated to $\sigma$, such that $s=\phi\left(\sigma, A^{\sigma}\right)(f)$.

Given a couple $\left(\sigma, A^{\sigma}\right)$ where $\sigma=\left(A_{i}\right)_{i \in I}$ is a measurable partition of $\Omega$, and $A^{\sigma}=\left\{a_{i}: i \in I\right\}$ is a finite set subordinated to $\sigma$, we consider $\psi\left(\sigma, A^{\sigma}\right)$, the application which maps each measurable correspondence $F: \Omega \rightarrow D$ to a simple measurable correspondence $\psi\left(\sigma, A^{\sigma}\right)(F)$, defined by

$$
\psi\left(\sigma, A^{\sigma}\right)(F):=\sum_{i \in I} F\left(a_{i}\right) \chi_{A_{i}} .
$$

Definition B.4. A correspondence $S: \Omega \rightarrow D$ is called a simple correspondence subordinated to a correspondence $F$ if there exists a couple $\left(\sigma, A^{\sigma}\right)$ where $\sigma$ is a measurable partition of $\Omega$, and $A^{\sigma}$ is a finite set subordinated to $\sigma$, such that $S=\psi\left(\sigma, A^{\sigma}\right)(F)$.

Remark B.5. If $f$ is a function from $\Omega$ to $D$, let $\{f\}$ be the correspondence from $\Omega$ into $D$, defined for each $a \in \Omega$ by $\{f\}(a):=\{f(a)\}$. We check that

$$
\psi\left(\sigma, A^{\sigma}\right)(F)=\left\{\phi\left(\sigma, A^{\sigma}\right)(f)\right\}
$$

\footnotetext{
${ }^{19}$ That is, for each $a \in \Omega, \chi_{A_{i}}(a)=1$ if $a \in A_{i}$ and $\chi_{A_{i}}(a)=0$ elsewhere.
} 
The space of all non-empty subsets of $D$ is noted $\mathcal{P}^{*}(D)$. We let $\tau_{W_{d}}$ be the Wijsman topology on $\mathcal{P}^{*}(D)$, that is the weak topology on $\mathcal{P}^{*}(D)$ generated by the family of distance functions $(d(x, .))_{x \in D \text {. }}$

Hereafter we assert that for a countable set of graph measurable correspondences, there exists a sequence of measurable partitions approximating each correspondence. The proof of the following theorem is given in Martins-da-Rocha [18].

Theorem B.6. Let $\mathcal{F}$ be a countable set of graph measurable correspondences with non-empty values from $\Omega$ into $D$ and let $\mathcal{G}$ be a finite set of integrable functions from $\Omega$ into $\mathbb{R}$. There exists a sequence $\left(\sigma^{n}\right)_{n}$ of finer and finer measurable partitions $\sigma^{n}=\left(A_{i}^{n}\right)_{i \in I^{n}}$ of $\Omega$, satisfying the following properties.

(a) Let $\left(A^{n}\right)_{n}$ be a sequence of finite sets $A^{n}$ subordinated to the measurable partition $\sigma^{n}$ and let $F \in \mathcal{F}$. For each $n \in \mathbb{N}$, we define the simple correspondence $F^{n}:=\psi\left(\sigma^{n}, A^{n}\right)(F)$ subordinated to $F$. Then for each $a \in \Omega, F(a)$ is the Wijsman limit of the sequence $\left(F^{n}(a)\right)_{n}$, i.e.,

$$
\forall a \in \Omega, \quad \forall x \in D, \quad \lim _{n} d\left(x, F^{n}(a)\right)=d(x, F(a)) .
$$

(b) There exists a sequence $\left(A^{n}\right)_{n}$ of finite sets $A^{n}$ subordinated to the measurable partition $\sigma^{n}$, such that for each $n$, if we let $f^{n}:=\phi\left(\sigma^{n}, A^{n}\right)(f)$ be the simple function subordinated to each $f \in \mathcal{G}$, then

$$
\forall f \in \mathcal{G}, \quad \forall a \in \Omega, \quad\left|f^{n}(a)\right| \leqslant 1+\sum_{g \in \mathcal{G}}|g(a)| .
$$

In particular, for each $f \in \mathcal{G}$,

$$
\lim _{n \rightarrow \infty} \int_{\Omega}\left|f^{n}(a)-f(a)\right| d \mu(a)=0 .
$$

Remark B.7. The property (a) implies in particular that, if $\left(x^{n}\right)_{n}$ is a sequence of $D, d$-converging to $x \in D$, then

$$
\forall a \in \Omega, \quad \lim _{n} d\left(x^{n}, F^{n}(a)\right)=d(x, F(a)) .
$$

It follows that if $F$ is non-empty closed valued, then property (a) implies that

$$
\forall a \in \Omega, \quad \operatorname{ls}_{n} F^{n}(a) \subset F(a) .
$$

\section{Appendix C. Fatou's Lemma}

The proof of the following theorem is given in Cornet-Martins-da-Rocha [10].

Theorem C.1. Let $(\Omega, \mathcal{A}, \mu)$ be a finite positive complete measure space. Let $\left(f_{n}\right)_{n}$ be a sequence of Bochner integrable mappings from $\Omega$ to $E_{+}$, which is mean norm-bounded, i.e.

$$
\sup _{n} \int_{\Omega}\left\|f_{n}(a)\right\| d \mu(a)<+\infty .
$$

Suppose that $w$-lim $\int_{\Omega} f_{n} d \mu$ exists in $E$ then there exists a Bochner integrable mapping $f$ such that

$$
\int_{\Omega} f d \mu \leqslant w-\lim _{n} \int_{\Omega} f_{n} d \mu
$$


and

$$
f(a) \in \overline{\operatorname{co}} w-\operatorname{ls}_{n}\left\{f_{n}(a)\right\} \quad \text { a.e. }
$$

Moreover

$$
\int_{\Omega}\|f(a)\| d \mu(a) \leqslant \sup _{n} \int_{\Omega}\left\|f_{n}(a)\right\| d \mu(a) .
$$

Appendix D. Fatou's COne

Proposition D.1. Let $(T, \mathcal{T}, \sigma)$ be an atomless measure space. For $1<p<+\infty$ we consider the space $E=L^{p}(T, \mathcal{T}, \sigma)$ ordered by the cone $E_{+}$defined by

$$
E_{+}=\left\{f \in L^{p}(T, \mathcal{T}, \sigma): \forall t \in T, f(t) \geqslant 0\right\} .
$$

The space $E$ ordered by the cone $E_{+}$is a Banach lattice. But for every Bochner integrable mapping $e: \Omega \rightarrow E_{+}$with $\int_{\Omega} e d \mu>0$, the cone $E_{+}$is not a Fatou's cone relatively to $e$.

Proof. Consider a Bochner integrable mapping $e: \Omega \rightarrow E_{+}$with $\int_{\Omega} e d \mu>0$. Suppose that $E_{+}$is a Fatou's cone relatively to $e$, then there exists a positive extended linear functional $\rho$ satisfying conditions (a) and (b) of Definition 2.2. Since $a \mapsto \rho[e(a)]$ is integrable, there exists $\Omega^{\prime}$ in $\mathcal{A}$ such that $\mu\left(\Omega \backslash \Omega^{\prime}\right)=1$ and for all $a \in \Omega^{\prime}, \rho[e(a)]<+\infty$. Moreover, since $\int_{\Omega} e d \mu>0$, there exists $b \in \Omega^{\prime}$ such that $e(b)>0$. We let $\omega=e(b)$, without any loss of generality, we may assume that $\|\omega\|=1$. We denote by $\delta$ the probability on $(T, \mathcal{T})$ defined by

$$
\forall B \in \mathcal{T}, \quad \delta(B)=\int_{B}[\omega(t)]^{p} \sigma(d t) .
$$

Since $(T, \mathcal{T}, \sigma)$ is atomless, the measure space $(T, \mathcal{T}, \delta)$ is also atomless. Applying Lyapunov Convexity Theorem (see Aliprantis-Border [2, Theorem 12.33]), $\{\delta(B): B \in \mathcal{T}\}=[0,1]$. Fix $n>0$, then there exists $T_{1} \in \mathcal{T}$ such that

$$
\int_{T_{1}} \omega^{p} d \sigma=\frac{1}{n}
$$

Now the restriction of $\delta$ to $T \backslash T_{1}$ is a finite positive measure, such that

$$
\left\{\delta(B): B \in \mathcal{T} \text { and } B \cap T_{1}=\emptyset\right\}=[0,1-1 / n] .
$$

Hence there exists $T_{2} \in \mathcal{T}$ with $T_{2} \cap T_{1}=\emptyset$ such that

$$
\int_{T_{2}} \omega^{p} d \sigma=\frac{1}{n}
$$

By induction, there exists a measurable partition $\left(T_{1}, \ldots, T_{n}\right)$ of $T$ such that

$$
\forall k \in\{1, \ldots, n\}, \quad \int_{T_{k}} \omega^{p} d \sigma=\frac{1}{n} .
$$

For each $k \in\{1, \ldots, n\}$, let $\chi_{k}$ be the characteristic function of $T_{k}$, i.e. $\chi_{k}(t)=1$ if $t \in T_{k}$ and $\chi_{k}(t)=0$ elsewhere. Then

$$
n^{1-1 / p}=\sum_{k=1}^{n}\left(\frac{1}{n}\right)^{1 / p}=\sum_{k=1}^{n}\left\|\omega \chi_{k}\right\| \leqslant \sum_{k=1}^{n} \rho\left[\omega \chi_{k}\right]=\rho(\omega),
$$

contradiction. 


\section{REFERENCES}

1. C. D. Aliprantis, Separable utility functions, Journal of Mathematical Economics 28 (1997), no. 4, 415-444.

2. C. D. Aliprantis and K. C. Border, Infinite dimensional analysis, Berlin: Springer, 1999.

3. A. Araujo and P. K. Monteiro, Equilibrium and dynamics: Essays in honor of David Gale, ch. General equilibrium with infinitely many goods: the case of separable utilities, Macmillan, London, 1989.

4. A. Araujo and P. K. Monteiro, Equilibrium without uniform conditions, Journal of Economic Theory 48 (1989), $416-427$.

5. K. J. Arrow and G. Debreu, Existence of an equilibrium for a competitive economy, Econometrica 22 (1954), 265-290.

6. R. J. Aumann, Markets with a continuum of agents, Econometrica 32 (1964), 39-50.

7. __ Existence of a competitive equilibrium in markets with a continuum of traders, Econometrica 34 (1966), $1-17$.

8. __ Measurable utility and measureable choice theorem, La Décision Centre National de la Recherche Scientifique, Paris (1969), 15-26.

9. T. F. Bewley, Equilibrium theory in infinite dimensional spaces, ch. A very weak theorem on the existence of equilibria in atomless economies with infinitely many commodities, Springer-Verlag: New-York, 1991.

10. B. Cornet and V. F. Martins-da-Rocha, Fatou's lemma for unbounded Gelfand integrable mappings, Working Paper, Université Paris-I Panthéon Sorbonne, 2002.

11. G. Debreu, Theory of value: An axiomatic analysis of economic equilibrium, New-York: John Wiley and Sons, 1959.

12. J. Diestel and J. Uhl, Vector measure, Mathematical Surveys, 1977.

13. M. Florenzano, On the non-emptiness of the core of a coalitional production economy without ordered preferences, Journal of Mathematical Analysis and Application 141 (1989), 484-490.

14. W. Hildenbrand, Existence of equilibria for economies with production and a measure space of consumers, Econometrica 38 (1970), 608-623.

15. L. E. Jones, Existence of equilibrium with infinitely many consumers and infinitely many commodities, Journal of Mathematical Economics 12 (1983), 119-138.

16. M. A. Khan and N. C. Yannelis, Equilibrium in markets with a continuum of agents and commodities, Equilibrium Theory in Infinite Dimensional Spaces (M. A. Khan and N.C. Yannelis, eds.), Berlin Heildelberg New-York: Springer-Verlag, 1991.

17. C. Le Van, Complete characterization of Yannelis-Zame and Chichilnisky-Kalman-Mas-Colell properness for separable concave functions defined in $L_{+}^{p}$ and $L^{p}$, Economic Theory 8 (1996), no. 1, 155-166.

18. V. F. Martins-da-Rocha, Existence of equilibria for economies with a measurable space of agents and non-ordered preferences, Working paper Université Paris-I Panthéon Sorbonne, 2001.

19. Equilibria in large economies with a separable Banach commodity space and non-ordered preferences, Journal of Mathematical Economics 39 (2003), no. 8, 863-889.

20. E__ Equilibria in large economies with differentiated commodities and non-ordered preferences, Economic Theory 23 (2004), no. 3, 529-552.

21. A. Mas-Colell, A model of equilibrium with differentiated commodities, Journal of Mathematical Economics 2 (1975), 263-295.

22. L. W. McKenzie, On equilibrium in Graham's model of world trade and other competitive systems, Econometrica 22 (1954), 147-161.

23. J. Ostroy and W. R. Zame, Nonatomic economies and the boundaries of perfect competition, Econometrica 62 (1994), 593-633.

24. K. Podczeck, Markets with infinitely many commodities and a continuum of agents with non-convex preferences, Economic Theory 9 (1997), 585-629.

25. _ Core and Walrasian equilibria when agents' characteristics are extremely dispersed, Economic Theory 22 (2003), no. 4, 699-725.

26. Note on the Core-Walras equivalence problem when the commodity space is a banach lattice, Working Paper \# 307, University of Vienna, 2003.

27. A. Rustichini and N. C. Yannelis, Edgeworth's conjecture in economies with a continuum of agents and commodities, Journal of Mathematical Economics 20 (1991), 307-326.

28. R. Tourky and N. C. Yannelis, Markets with many more agents than commodities: Aumann's "hidden" assumption, Journal of Economic Theory 101 (2001), 189-221. 
29. N. C. Yannelis and W. R. Zame, Equilibria in Banach lattices without ordered preferences, Journal of Mathematical Economics 15 (1986), 75-110.

30. W. R. Zame, Markets with a continuum of traders and infinitely many commodities, Working paper, SUNY at Buffalo, 1986.

Instituto Nacional de Matemtica Pura e Aplicada - impa and EPGE/FGV, Estrada Dona Castorina 110, CEP 22460, Rio de Janeiro, RJ, BraziL

E-mail address: aloisio@impa.br

Ceremade, Univerisité Paris-IX Dauphine, Place du Marchal de Lattre de Tassigny, 75775 Paris Cedex 16, France

E-mail address: martins@ceremade.dauphine.fr

EPGE/FGV - Praia de Botafogo 190 - 11 andar Botáfogo, 22250-900 Rio de Janeiro, RJ, BraziL E-mail address: PKLM@fgv.br 